\title{
Adverse Maternal Consequences Associated with Prolonged Acute-Onset Severe Systolic Hypertension during Pregnancy \& Early Postpartum: Pitfalls in Practice \& Lessons Learned
}

\author{
James Nello Martin Jr. ${ }^{1}$, James Martin Tucker ${ }^{2}$ \\ ${ }^{1}$ Obstetrics, Gynecology \& Maternal-Fetal Medicine, The Winfred L. Wiser Hospital for Women \& Infants, The University of \\ Mississippi Medical Center, Jackson, Mississippi, USA \\ ${ }^{2}$ Obstetrics \& Gynecology, The Winfred L. Wiser Hospital for Women \& Infants, The University of Mississippi Medical Center, \\ Jackson, Mississippi, USA \\ Email: jnmartinjrmd@gmail.com
}

How to cite this paper: Martin Jr., J.N. and Tucker, J.M. (2021) Adverse Maternal Consequences Associated with Prolonged Acute-Onset Severe Systolic Hypertension during Pregnancy \& Early Postpartum: Pitfalls in Practice \& Lessons Learned. Open Journal of Obstetrics and Gynecology, 11, 626-635.

https://doi.org/10.4236/ojog.2021.115058

Received: April 25, 2021

Accepted: May 24, 2021

Published: May 27, 2021

Copyright $\odot 2021$ by author(s) and Scientific Research Publishing Inc. This work is licensed under the Creative Commons Attribution International License (CC BY 4.0).

http://creativecommons.org/licenses/by/4.0/ (c) (i) Open Access

\begin{abstract}
OBJECTIVE: To determine the types of major maternal-perinatal morbidity associated with prolonged, acute-onset severe systolic hypertension during pregnancy and postpartum. METHODS: A medicolegal database retaining only medical record data was created from all cases involving women with medical/hypertensive disorders of pregnancy evaluated by the first author between 1986-2015. Case files of women that experienced severe systolic hypertension (SSH) sustained for many hours to days were identified for study. RESULTS: Sixty six pregnant/postpartum women met study criteria. Stroke secondary to intracranial hemorrhage or thrombosis (65.2) and acute pulmonary edema $(33 \%)$ were the leading causes of maternal morbidity and mortality, most often antepartum as a component of early-onset preeclampsia ( $\leq 34$ weeks). Eclampsia, abruptio placenta and injury to heart, liver and/or kidneys were other frequent co-morbidities. Seven postpartum women developed sudden new-onset postpartum SSH and suffered a stroke 4 - 13 days after delivery. Maternal mortality (54.6\%) and morbidity as persistent disability $(24.2 \%)$ were high in this cohort. CONCLUSION: Failure to rapidly respond, reduce and sustain at a safe level acute-onset SSH poses a significant threat to the wellbeing of mothers and babies, before and in the weeks following delivery. Systems to implement safe practices to identify and emergently treat severe maternal hypertension are needed.
\end{abstract}




\section{Keywords}

Severe Systolic Hypertension Acute Pulmonary Edema Maternal Morbidity \& Mortality Maternal Safety

\section{Introduction}

One of the most significant recent advances in obstetric practice is the maternal safety campaign to coordinate efforts by health care professionals to recognize and respond rapidly to any peripartum patient who develops acute-onset sustained severe systolic and/or diastolic hypertension (SSH) [1] [2] [3] [4] [5]. Efforts are focused primarily toward reduction of the mother's risk for stroke. Based largely upon experience accrued via medicolegal reviews from venues where obstetrics and gynecology specialists were providing perinatal care to 21 patients in non-teaching medical centers, supplemented with 7 additional cases managed at the University of Mississippi Medical Center, investigators in 2004 made several important observations. Data analyzed from these 28 women revealed that exceeding/sustaining a systolic blood pressure range above a threshold between 155 - $160 \mathrm{mmHg}$ (and not diastolic hypertension) preceded every stroke in patients with acute-onset hypertensive disorders of pregnancy [1]. This novel finding was consistent with findings published in 1995 showing that systolic blood pressure in adults is a more robust stroke predictor in adults than diastolic pressures [6]. Recently others reported similar findings from a cohort of preeclampsia-related deaths in California between 2002-2007 [7].

The duration of SSH sustained before stroke occurred was not studied nor were patient outcomes assessed with regard to other obstetric complications or injury to other maternal organ systems. We believe that aggregated medicolegal reviews focused on selected infrequent but important issues, similar to maternal mortality reviews, can provide worthwhile information to assess infrequent adverse outcomes that occur in relation to other clinical events. Our purpose in this investigation is to explore in a large patient database the broad impact of acute-onset sustained prolonged SSH upon the mother and her fetus, during pregnancy and the early postpartum period.

\section{Methods}

Among the 161 medical or hypertensive disorders of pregnancy-related cases the first author reviewed for medicolegal defense purposes between 1988-2016, there were 66 women that met inclusion criteria for sudden-onset, sustained severe hypertension (at least two severe blood pressures, systolic $\geq 160 \mathrm{mmHg}$ and/or diastolic $\geq 110 \mathrm{mmHg}$, confirmed as persistent for $\geq 15$ minutes) [8] for hours to days during late pregnancy and/or postpartum. Clinical summaries were prepared by the first author from the case medical records and fact-checked by the second author; data sheets structured for later analysis included all available 
medical record information including details of patient presentation, significant clinical findings, lab results, surgeries, interventions, complications, and final outcomes as illustrated in the tables shown. All other materials related to legal matters and communications had been shredded years earlier after case resolution and the confidential residual medical records thereafter kept secure in order to meet ethical and HPPA guidelines.

Findings from this analysis are grouped into five chronologic categories according to the timing of major adverse maternal event(s) related to SSH: ANTEPARTUM, INTRAPARTUM, POSTPARTUM following delivery while hospitalized, DELAYED POSTPARTUM following hospital discharge, and a fifth "NO EVENT" category without adverse effects associated with or attributable to $\mathrm{SSH}$. Main categories of adverse events included intracranial hemorrhage, cerebral thrombosis, eclampsia/eclampsia-like convulsion, placental abruption, acute pulmonary edema, acute kidney injury, acute liver rupture/hemorrhage, and cardiac disorder.

\section{Results}

Data fields could be completed for 64 of 66 study subjects; partial data was available for the other two women, thus excluding them from most calculations. Pregnancy and postpartum care spanned the years 1988-2016. Maternal age ranged from 17 - 43 years. Only 1 of 12 women in the intrapartum group was older than 30 , whereas the mean age for the other groups was $\geq 30$ years ranging from $43 \%$ (3/7) for the delayed postpartum group to $65.4 \%$ (17/26) for the antepartum group. Maternal weights ranged between $110-330$ pounds ( mean $=183$ lbs); 16 of 59 weighed $\geq 200$ lbs. Mean gestational age for all groups was 34.6 weeks; two thirds of women with antepartum or intrapartum events happened $\leq 34$ weeks whereas almost all delayed postpartum event women had full term pregnancies (mean 38.5 weeks). Almost half $(47.7 \%)$ of the women in the combined cohort were White, $36.9 \%$ were Black, $13.8 \%$ were Hispanic and one was Asian. More than half were nulliparous; every woman in the delayed postpartum group was multiparous.

Relationships among the five SSH groups versus the types of major adverse events are tabulated in Table 1. Stroke secondary to intracranial hemorrhage (ICH) or thrombosis caused most of the major maternal adverse events associated with SSH around the time of delivery. When ICH was neuroimaged, multiple affected areas were usually detected most commonly involving the basal ganglia, parietal and frontal areas. Although 13 eclamptic convulsions were described among the 66 women cohort, 7 were eventually determined to have occurred in association and concurrent with ICH or thromboses. One of the six eclamptic convulsions occurring independent of ICH happened with a placental abruption, whereas another occurred as the patient developed acute pulmonary edema.

The peak systolic blood pressure recorded prior to an adverse event ranged 
Table 1. Major adverse maternal \& perinatal events associated with severe systolic hypertension.

\begin{tabular}{|c|c|c|c|c|c|c|c|c|}
\hline GROUP & $\mathrm{ICH}$ & CVT & Eclampsia & Abruptio & Lung & Kidney & Heart & Liver \\
\hline Antepartum $(\mathrm{n}=27 ; 41 \%)$ & 21 & 1 & 7 & 3 & 9 & 4 & 3 & 3 \\
\hline Intrapartum $(\mathrm{n}=12 ; 18.2 \%)$ & 1 & - & 2 & 7 & 4 & 2 & - & - \\
\hline Postpartum $(n=16 ; 24.2 \%)$ & 12 & 1 & 2 & - & 7 & 2 & 1 & 2 \\
\hline Delayed PP $(n=7 ; 10.6 \%)$ & 6 & 1 & 1 & - & 2 & - & - & - \\
\hline None $(\mathrm{n}=4 ; 6.1 \%)$ & - & - & 1 & - & - & - & - & - \\
\hline TOTALS $(\mathrm{n}=66)$ & 40 & 3 & 13 & 10 & 22 & 8 & 4 & 5 \\
\hline PERCENTAGE of TOTAL & $61 \%$ & $4.6 \%$ & $19.7 \%$ & $15.2 \%$ & $33 \%$ & $12 \%$ & $6 \%$ & $7.6 \%$ \\
\hline
\end{tabular}

In the table, $\mathrm{ICH}=$ intracranial hemorrhage, $\mathrm{CVT}=$ cerebral venous thrombosis, $\mathrm{PP}=$ postpartum.

between 160 - $243 \mathrm{mmHg}$ with similar mean value for all groups (187 - 193 $\mathrm{mmHg}$ ). The peak diastolic blood pressure recorded prior to an adverse event varied, ranging from $88 \mathrm{mmHg}$ to $147 \mathrm{~mm}$ with similar mean values for all groups (107 - $113 \mathrm{mmHg}$ ). Because duration of sustained severe hypertension may be as important as level of severity, this issue was explored in 20 patients for whom data was available; 14 were related to stroke (range 1.5 to 120 continuous hours before stroke occurred, mean 32.5 hours) and 6 preceded placental abruption, acute pulmonary edema and eclampsia (range 0.5 to 11 days, mean 6.3 days). Even though SSH was present in 17 of 65 women $(26.2 \%)$, less than a third received antihypertensive therapy prior to an adverse event and in no case was it controlled (systolic $\leq 160 \mathrm{mmHg}$ sustained). Only 31 of 65 (47.7\%) received magnesium sulfate.

Severe epigastric/upper right quadrant pain occurred only in the ANTEPARTUM, INTRAPARTUM and IMMEDIATE POSTPARTUM patient groups. Seventeen of 18 ANTEPARTUM patients, 2 of 7 INTRAPARTUM patients and all 8 of the IMMEDIATE POSTPARTUM women with epigastric pain developed HELLP syndrome. The time course from onset of severe epigastric pain to adverse event could be determined only for three women: 3 - 4 hours for two women that did not receive fetal lung maturation steroids versus 5 days for one woman that did [9] [10].

All women having a cerebral event reported severe headache. Headache described as severe that preceded ICH was recorded for 20 of $23(87 \%)$ women in the ANTEPARTUM event group, 14 of 16 (87.5\%) women in the IMMEDIATE POSTPARTUM event group, and all 6 (100\%) of the DELAYED POSTPARTUM event group. Eight of 12 women in the INTRAPARTUM event group had severe headache, one suffering an ICH. The time course from onset of severe headache until adverse event could be determined for five women: 6 - 48 hours for 3 women not receiving fetal lung maturation steroids versus 36 hours to 9 days for two women who did. Among women with SSH who presented in the ANTEPARTUM, INTRAPARTUM and POSTPARTUM event groups, there were 26 women who met criteria to receive antepartum corticosteroids for fetal indi- 
cations. [11] Only 10 of 26 (38.5\%), however, received steroid therapy. Subsequent adverse events that occurred thereafter as stroke $(n=5)$, placental abruption $(n=2)$ or acute pulmonary edema $(n=3)$ occurred in most women $(80 \%) \geq$ 24 hours following only the second of the two-injection steroid regimen. The 7 DELAYED POSTPARTUM women had stroke events at home or in route back to the hospital on postpartum days $4,5,5,6,6,7$ and 13. Three died and four were permanently disabled.

Mode of delivery was cesarean in 49 of 65 (75.4\%) women. Table 2 illustrates eventual maternal outcome for the five patient groups regarding maternal mortality $(n=36)$, permanent maternal disability $(n=16)$, or intact maternal survival $(n=14)$. Altogether almost $80 \%$ of the women in this select medicolegal cohort suffered permanent injury or death following prolonged sustained SSH. Perinatal outcomes included 6 stillbirths, 5 neonatal deaths, 9 newborn non-intact survivors and 44 intact newborns.

\section{Discussion}

In this select cohort of women that experienced prolonged, sustained SSH, maternal stroke was the most common type of major adverse event resulting in major maternal morbidity and/or maternal mortality. Beyond stroke, there are other potentially significant sequelae to uncontrolled SSH [12] which include threats to lung, heart [13], liver and kidney tissues in addition to placental abruption and eclamptic convulsion. The very high maternal mortality and morbidity as permanent disability are compelling reasons to minimize possible adverse effects of SSH by responding emergently with effective measures as recommended since late 2011 by ACOG [14] [15] [16]. With the goal of reducing maternal morbidity and mortality in the United States, the Joint Commission for Hospital Accreditation has recently mandated the integration of safety bundles and practice guidelines in hospitals providing care to obstetric patients with severe hypertension [17]. The shortest interval recorded from onset of SSH to stroke occurrence was 90 minutes in our study cohort, supporting the current recommendation to recognize and treat within 60 minutes of verifying sustained severe hypertension.

Acute pulmonary edema with respiratory compromise also can develop in the

Table 2. Maternal adverse outcomes subsequent to sustained severe hypertension.

\begin{tabular}{cccc}
\hline & Deaths & Disability & Healthy Outcome \\
\hline ANTEPARTUM Group $(\mathrm{n}=27)$ & 20 & 5 & 2 \\
INTRAPARTUM Group $(\mathrm{n}=12)$ & 3 & 2 & 7 \\
POSTPARTUM Group $(\mathrm{n}=16)$ & 10 & 6 & 0 \\
DELAYED POSTPARTUM Group $(\mathrm{n}=7)$ & 3 & 3 & 1 \\
No Adverse Event Group $(\mathrm{n}=4)$ & 0 & 0 & 4 \\
TOTALS $(\mathrm{n}=66)$ & $36(54.6 \%)$ & $16(24.2 \%)$ & $14(21.2 \%)$ \\
\hline
\end{tabular}


setting of prolonged sustained acute-onset severe maternal hypertension either before or following delivery. Acute pulmonary edema is well known to occur in approximately $3 \%$ of patients with severe forms of preeclampsia spectrum disorders, most frequently seen in older, multigravid patients [18] although its development is multifactorial [19] [20] [21] [22]. Among the four patient groups developing adverse events associated with SSH, one third (22 of 66) experienced acute pulmonary edema most likely to occur around the time of delivery [23]. Maternal heart disease (4 women) often accompanied findings of acute pulmonary edema, further challenging patient management. Clinicians must remain alert to detect signs of maternal cardiopulmonary compromise when SSH occurs.

Certainly a contributing factor in our findings is that only 19 of our 39 women (48.7\%) with adverse antepartum or intrapartum events received magnesium sulfate and only $12(30.8 \%)$ received antihypertensives. The infrequency of these therapies in many patients reviewed for this series is an argument for widespread utilization of comprehensive practice guidelines and safety bundles for health care professionals-nurses and physicians-to help enhance systematic patient care and safety before and after delivery when complications such as SSH develop [24] [25].

Severe epigastric pain caused by developing HELLP syndrome [26] [27] and SSH appear to be closely related; aggressive treatment of one likely will benefit the other. In our patient cohort, 27 of 33 (82\%) women with epigastric pain and SSH were found to have HELLP syndrome. A critical window between onset of epigastric pain and stroke happened in as few as 3 hours. Two therapies for effective alleviation of epigastric pain in patients with HELLP syndrome are magnesium sulfate alone or combined therapy using magnesium sulfate with a glucocorticoid such as dexamethasone [28] [29]. Maternally administered corticosteroids indicated for fetal purposes also appear to have a TRANSIENT (24 - 48 hours) beneficial maternal impact upon maternal hypertension, delay of epigastric pain onset and developing HELLP syndrome. Less than half or 10 of 26 mothers who were appropriate candidates to receive steroids for fetal benefit actually received treatment. When indicated, a steroid course should be initiated without delay especially in at-risk patients like those described in this group of women with SSH [30]. Similar to other reports, at least four patients in this cohort demonstrated a temporary reduction in the severity of systolic hypertension up to 48 hours following the maternal receipt of a fetal lung maturation steroid course [31].

Although this patient series is large, we acknowledge several shortcomings, the most important of which is the risk of selection bias in any undertaking involving a group of pregnant and postpartum patients which seeks medicolegal relief and compensation associated with adverse maternal and/or perinatal outcome. Were more data retrievable relative to exact time of event onset and subsequent outcomes for more or most of the cases, a stronger case could be made for likely associations as cause and effect. We have no way to construct a suitable 
control group of patients for comparison purposes.

Finally, we note that SSH can develop after delivery discharge without warning, pointing toward a consideration to evaluate enhanced remote universal surveillance of blood pressure for all postpartum patients. As shown by Gloria Too and colleagues using data from the Nationwide Readmissions Database, the majority of stroke readmissions occur within 10 days of hospital discharge often in the absence of known prior hypertension [32].

Inadequate or delayed response to acute-onset SSH hopefully will soon disappear as a reportable event in maternal quality and safety monitoring programs in the United States. This should happen as more obstetric hypertensive emergency quality improvement initiatives are undertaken and extensive educational undertakings are implemented [33]. The search for safer motherhood around this issue will happen only when best practice is integrated into systems of care for health care professionals to provide to all maternity patients [34] [35].

\section{Confirmed}

Each author has confirmed compliance with the journal's requirements for authorship. The authors received no financial support for this undertaking. Neither has any potential conflict of interest. The first author prepared the original manuscript which the second author critiqued. This information has not been presented to a professional meeting nor is it under consideration for publication elsewhere.

\section{Conflicts of Interest}

The authors declare no conflicts of interest regarding the publication of this paper.

\section{References}

[1] Martin, J.N., Thigpen, B.D., Moore, R.C., Rose, C.H., Cushman, J. and May, W. (2005) Stroke and Severe Preeclampsia and Eclampsia: A Paradigm Shift Focusing on Systolic Blood Pressure. Obstetrics \& Gynecology, 105, 246-254. https://doi.org/10.1097/01.AOG.0000151116.84113.56

[2] ACOG Committee Opinion No. 514 (2011) Emergent Therapy for Acute-Onset, Severe Hypertension during Pregnancy and the Postpartum Period.

[3] ACOG Committee Opinion No. 767 (2019) Emergent Therapy for Acute-Onset, Severe Hypertension during Pregnancy and the Postpartum Period. Obstetrics \& Gynecology, 133, 409-412.

[4] (2013) Hypertension in Pregnancy. Report of the ACOG Task Force on Hypertension in Pregnancy. Obstetrics \& Gynecology, 122, 1122-1131. https://doi.org/10.1097/01.AOG.0000437382.03963.88

[5] Gupta, M., Greene, N. and Kilpatrick, S.J. (2018) Timely Treatment of Severe Maternal Hypertension and Reduction in Severe Maternal Morbidity. Pregnancy Hypertension, 14, 55-58. https://doi.org/10.1016/j.preghy.2018.07.010

[6] Lindenstrom, E., Boysen, G. and Nyboe, J. (1995) Influence of Systolic and Diastolic Blood Pressure on Stroke Risk: A Prospective Observational Study. American Jour- 
nal of Epidemiology, 142, 1279-1290.

https://doi.org/10.1093/oxfordjournals.aje.a117595

[7] Judy, A.E., McCain, C.L., Lawton, E.S., Morton, C.H., Main, E.K. and Druzin, M.L. (2019) Systolic Hypertension, Preeclampsia-Related Mortality, and Stroke in California. Obstetrics \& Gynecology, 133, 1151-1159. https://doi.org/10.1097/AOG.0000000000003290

[8] ACOG Practice Bulletin 222 (2020) Gestational Hypertension and Preeclampsia. Obstetrics \& Gynecology, 135, e237-e260. https://doi.org/10.1097/AOG.0000000000003891

[9] Raymond, D. and Peterson, E. (2011) A Critical Review of Early-Onset and LateOnset Preeclampsia. Obstetrical \& Gynecological Survey, 66, 497-506.

https://doi.org/10.1097/OGX.0b013e3182331028

[10] Lisonkova, S., Sabr, Y., Mayer, C., Young, C., Skoll, A. and Joseph, K.S. (2014) Maternal Morbidity Associated with Early-Onset and Late-Onset Preeclampsia. Obstetrics \& Gynecology, 124, 771-781. https://doi.org/10.1097/AOG.0000000000000472

[11] ACOG Committee Opinion 713 (2017) Antenatal Corticosteroid Therapy for Fetal Lung Maturation. Obstetrics \& Gynecology, 130, e102-e109.

https://doi.org/10.1097/AOG.0000000000002237

[12] ElFarra, J., Bean, C. and Martin, J.N. (2016) Management of Hypertensive Crisis for the Obstetrician/Gynecologist. Obstetrics and Gynecology Clinics of North America, 43, 623-637. https://doi.org/10.1016/j.ogc.2016.07.005

[13] Vaughn, A.J., Kovell, L.C., Szymanski, L.M., Mayer, S.A., Seifert, S.M., et al. (2018) Acute Cardiac Effects of Severe Preeclampsia. Journal of the American College of Cardiology, 72, 1-11. https://doi.org/10.1016/j.jacc.2018.04.048

[14] Cantwell, R., Clutton-Brock, T., Cooper, G., et al. (2011) Saving Mothers' Lives: Reviewing Maternal Deaths to Make Motherhood Safer: 2006-2008. The Eighth Report of the Confidential Inquiries into Maternal Deaths in the United Kingdom. British Journal of Obstetrics and Gynaecology, 118, 1-203. https://doi.org/10.1111/j.1471-0528.2010.02847.x

[15] Clark, S.L., Christmas, J.T., Frye, D.K., Meyers, J.A., Frye, D.K. and Perlin, J.B. (2014) Maternal Mortality in the United States: Predictability and the Impact of Protocols on Fatal Postcesarean Pulmonary Embolism and Hypertension-Related Intracranial Hemorrhage. American Journal of Obstetrics and Gynecology, 211, 32.e1-e9. https://doi.org/10.1016/j.ajog.2014.03.031

[16] Bushnell, C., McCullough, L.D., Awad, I.A., et al. (2014) Guidelines for the Prevention of Stroke in Women: A Statement for the Healthcare Professionals from the American Heart Association/American Stroke Association. Stroke, 45, 1545-1588. https://doi.org/10.1161/01.str.0000442009.06663.48

[17] Joint Commission Standards PC.06.01.01 \& PC.0601.03 Effective July 1, 2020 Standards Addressing Prevention, Early Recognition and Timely Treatment of Maternal Hemorrhage and Severe Hypertension/Preeclampsia.

https://www.jointcommission.org

[18] Sibai, B.M., Mabie, B.C., Harvey, C.J. and Gonzalez, A.R. (1987) Pulmonary Edema in Severe Preeclampsia-Eclampsia: An Analysis of Thirty-Seven Consecutive Cases. American Journal of Obstetrics and Gynecology, 156, 1174-1179. https://doi.org/10.1016/0002-9378(87)90135-9

[19] Bauer, S.T. and Cleary, K.L. (2009) Cardiopulmonary Complications of Pre-Eclampsia. Seminars in Perinatology, 33, 158-165.

https://doi.org/10.1053/j.semperi.2009.02.008 
[20] Ford, L.E. (2010) Acute Hypertensive Pulmonary Edema: A New Paradigm. Canadian Journal of Physiology and Pharmacology, 88, 9-13. https://doi.org/10.1139/Y09-113

[21] Thornton, C.E., von Dadelszen, P., Makris, A., Tooher, J.M., Ogle, R.F. and Hennessy, A. (2011) Acute Pulmonary Oedema as a Complication of Hypertension during Pregnancy. Hypertension in Pregnancy, 30, 169-179. https://doi.org/10.3109/10641950902972140

[22] Figueras, J., Baneras, J., Pena-Gil, C., Masip, J., Barrabes, J.A., Palomares, J.R. and Garcia-Dorado, D. (2016) Acute Arterial Hypertension in Acute Pulmonary Edema: Mostly a Trigger or an Associated Phenomenon. The Canadian Journal of Cardiology, 32, 1214-1220. https://doi.org/10.1016/j.cjca.2015.10.030

[23] Pordeus, A.C., Katz, L., Soares, M.C., Mala, S.B. and Amorim, M.M. (2018) Acute Pulmonary Edema in an Obstetric Intensive Care Unit: A Case Series Study. Medicine (Baltimore), 97, e11508. https://doi.org/10.1097/MD.0000000000011508

[24] Bernstein, P.S., Martin, J.N., Barton, J.R., Shields, L.E., Druzin, M.L., Scavone, B.M., Frost, J., Morton, C.H., Ruhl, C., Slager, J., Tsigas, E.Z., Gaffer, S. and Menard, M.K. (2017) National Partnership for Maternal Safety: Consensus Bundle on Severe Hypertension during Pregnancy and the Postpartum Period. Obstetrics \& Gynecology, 130, 347-357. https://doi.org/10.1097/AOG.0000000000002115

[25] Hogg, J.P. III, Szczepanski, J.L., Collier, C. and Martin, J.N. (2020) Immediate Postpartum Management of Patients with Severe Hypertensive Disorders of Pregnancy: Pathophysiology Guiding Practice. Journal of Maternal-Fetal and Neonatal Medicine, 10, 1-11. https://doi.org/10.1080/14767058.2020.1776251

[26] Martin, J.N., Rinehart, B.K., May, W.L., Magann, E.F., Terrone, D.A. and Blake, P.G. (1999) The Spectrum of Severe Preeclampsia: Comparative Analysis by HELLP (Hemolysis, Elevated Liver Enzyme Levels, and Low Platelet Count) Syndrome Classification. American Journal of Obstetrics and Gynecology, 180, 1373-1384. https://doi.org/10.1016/S0002-9378(99)70022-0

[27] Suarez, B., Alves, K., Senat, M.V., Fromageot, J., Fischer, C., Rosenberg, P. and Ville, Y. (2002) Abdominal Pain and Preeclampsia: Sonographic Findings in the Maternal Liver. Journal of Ultrasound in Medicine, 21, 1077-1083. https://doi.org/10.7863/jum.2002.21.10.1077

[28] Martin, J.N., Owens, M.Y.O., Keiser, S.D., Parrish, M.R., Tam Tam, K.B., Brewer, J.M., Cushman, J.L. and May, W.L. (2012) Standardized Mississippi Protocol Treatment of 190 Patients with HELLP Syndrome: Slowing Disease Progression and Preventing New Major Maternal Morbidity. Hypertension in Pregnancy, 31, 79-90. https://doi.org/10.3109/10641955.2010.525277

[29] Wallace, K., Hrris, S., Addison, A. and Bean, C. (2018) HELLP Syndrome: Pathophysiology and Current Therapies. Current Pharmaceutical Biotechnology, 19, 816826. https://doi.org/10.2174/1389201019666180712115215

[30] Young, C.M., Schneider, P., Nelson, L. and Julien, S. (2015) Outcomes of Expectant Management after Betamethasone for Hypertensive Disorders of Pregnancy. Hypertension in Pregnancy, 34, 50-64.

https://doi.org/10.3109/10641955.2014.966912

[31] Nayeri, U.A., Buhimschi, I.A., Laky, C.A., Cross, S.N., Duzyi, C.M., Ramma, W., Sibai, B.M., Funai, E.F., Ahmed, A. and Buhimschi, C.S. (2014) Antenatal Corticosteroids Impact the Inflammatory Rather than the Antiangiogenic Profile of Women with Preeclampsia. Hypertension, 63, 1285-1292.

https://doi.org/10.1161/HYPERTENSIONAHA.114.03173 
[32] Too, G., Wen, T., Boehme, A.K., Miller, E.C., Leffert, L.R., Attenello, F.J., Mack, W.J., D’Alton, M.E. and Friedman, A.M. (2018) Timing and Risk Factors of Postpartum Stroke. Obstetrics \& Gynecology, 131, 70-78.

https://doi.org/10.1097/AOG.0000000000002372

[33] Froehlich, R.J., Maggio, L., Has, P., Vrees, R. and Hughes, B.L. (2018) Improving Obstetric Hypertensive Emergency Treatment in a Tertiary Care Women's Emergency Department. Obstetrics \& Gynecology, 132, 850-858.

https://doi.org/10.1097/AOG.0000000000002809

[34] Friedman, A.M., Campbell, M.L., Kline, C.R., Wiesner, S., D’Alton, M.E. and Shields, L.E. (2018) Implementing Obstetric Early Warning Systems. AJP Reports, 8, e79e84. https://doi.org/10.1055/s-0038-1641569

[35] Shields, L.E., Wiesner, S., Klein, C., Pelletreau, B. and Hedriana, H.L. (2017) Early Standardized Treatment of Critical Blood Pressure Elevations Is Associated with a Reduction in Eclampsia and Severe Maternal Morbidity. American Journal of $O b$ stetrics and Gynecology, 216, 415.e1-415.e5.

https://doi.org/10.1016/j.ajog.2017.01.008 